\title{
A Triaxial Force Discernment Algorithm for Flexible, High Density, Artificial Skin
}

\author{
Seth Koterba and Yoky Matsuoka \\ Robotics Institute \\ Carnegie Mellon University \\ Pittsburgh, PA 15213 \\ Email: skoterba@cs.cmu.edu yoky@cs.cmu.edu
}

\begin{abstract}
Artificial skin research has focused on three primary characteristics: high density, flexible sensing arrays, and the ability to decompose all three components of an applied force vector. Previous research has accomplished some but not all of these characteristics. In this paper, we present a new flexible, high density sensor array and a triaxial force discernment algorithm that can be used with commercially available flexible high density sensor arrays. Together, the sensing array and algorithm achieve all three of the critical skin characteristics. With an addition of thin silicone pillar arrays, the triaxial force discernment algorithm provides a direct solution for nearly all possible force directions. Where the solution is ambiguous, our algorithm is able to reduce the uncertainty to a small range of known values. To confirm the validity of the algorithm, a testbed for a single sensing element was developed. Initial results show a resolution of less than 0.01 radians and calculated force components within the uncertainty of the ground truth for most situations. Our approach will open doors for many applications including robotic manipulation, haptic interfaces, human-robot interaction, and medical diagnoses.
\end{abstract}

Index Terms - artificial skin, high density tactile sensors, flexible, pillar structure, triaxial force discernment.

\section{INTRODUCTION}

Replicating the force sensing capabilities of the human skin in a pliable material has never been accomplished. Developing such a material has been a goal of engineering research in areas such as robotic manipulation, haptic interfaces, human-robot interaction, and medical diagnoses. For example, achieving dextrous manipulation or exploration of the environment using a robotic hand requires detailed knowledge of contact forces between a manipulator and the interacting object. In the area of human-robot interaction, high density tactile sensors on the robot would provide awareness of their environment and could prevent human injury. Currently available sensors lack one or more of these features: high receptor density, multi-dimensional force discernment, and a flexible sensing array that can conform to arbitrarily shaped object, bodies or end effectors [1-8].

Yamada, Maeno and Yamada [1] have pioneered the curved fingertip design, and have also worked with artificial ridges, which aid in discerning normal and tangential forces, giving biaxial force information. Papakostas, Lima, and Lowe [2] have designed a high spatial resolution sensor based on piezoresitive technology, which is thin and highly flexible. This sensor array is ideal for draping over curved surfaces, but is unable to differentiate force components. Kane, Cutkosky and Kovacs [3] and Liu, Wang and Beebe [4] have developed techniques that allow high resolution and discrimination of the three components of an applied force. However, the drawback of these designs is that the sensing arrays are etched into flat rigid substrates. These rigid designs hinder the possibility of conforming to arbitrary shapes. Hakozaki and Shinoda [5] also presented a skin design based on microelectronics. Three millimeter wide rigid LSI chips are embedded in a rubbery substrate at intervals of several millimeters to allow flexibility. While each chip is able to determine three components of the stress within the substrate, the high receptor density is traded off with the flexibility of the artificial skin. A summary of these designs with respect to the three crucial features is shown in Table I.

TABLE I

COMPARISON OF ARTIFICIAL SKIN DESIGNS

\begin{tabular}{|l|c|c|c|}
\hline \multicolumn{1}{|c|}{ Group } & Flexibility & $\begin{array}{c}\text { High Receptor } \\
\text { Density }\end{array}$ & $\begin{array}{c}\text { Dimensionality } \\
\text { of Force } \\
\text { Discernment }\end{array}$ \\
\hline Yamada et al. & Yes & No & 2 \\
\hline Papakostas et al. & Yes & Yes & 1 \\
\hline Kane et al. & No & Yes & 3 \\
\hline Liu et al. & No & Yes & 3 \\
\hline Hakozaki et al. & Yes & No & 3 \\
\hline
\end{tabular}

This paper introduces a new flexible and high density sensing array and an algorithm that provides triaxial (i.e. three independent components) force discernment of an applied force for this array. Section II provides the design of the sensing array prototype and Section III presents the triaxial force discrimination algorithm. Sections IV and V describe the construction of a single sensing element and initial results that validate the algorithm. Finally, Section VI concludes with a discussion and proposal of future work.

\section{SKIN DESIGN}

Our skin design incorporates and adapts some of the techniques that have proven successful in $[1,2,4]$. The backbone of the skin design is Tekscan's piezoresitive technology, which is thin, flexible, allows high resolution arrays, and provides excellent sensitivity. Our prototype high density array design incorporates Tekscan's \#5027 sensor which provides a pressure resolution better than $0.02 \mathrm{~kg} / \mathrm{cm}^{2}$ and spatial resolution of 250 "sensels" per square centimeter [6]. Tekscan sensors alone, however, do not provide three separate components of the applied force. To solve this, we added an array of small polydimethylsiloxane (PDMS) pillars on top of the sensing array, as shown in Fig. 1. Any force 


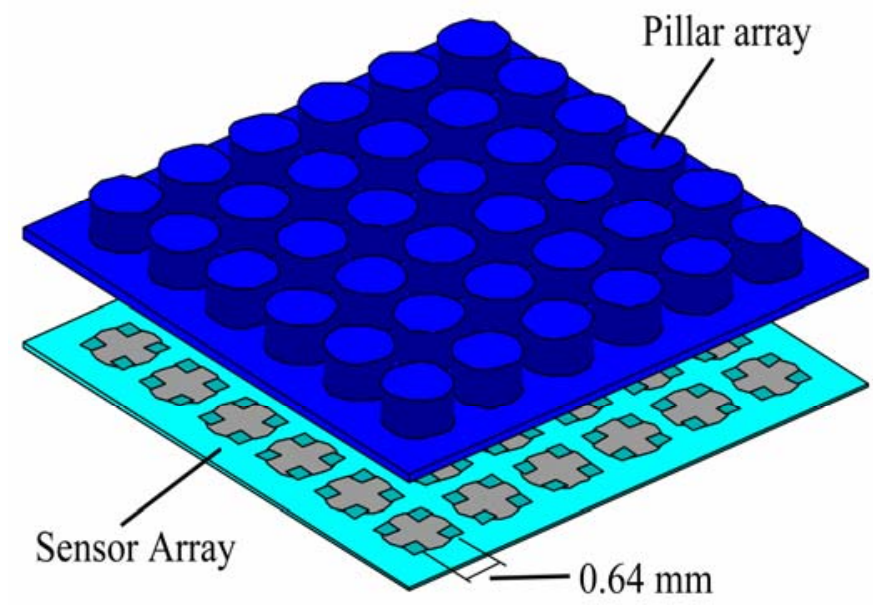

Fig. 1 Sketch of the artificial skin design. The sensing elements are small square cells on the sensor array. Shaded outlines of the pillars are drawn on the sensor to visualize the alignment of the two arrays.

applied to the top of the pillar is directed down through the base of the pillar and is concentrated around the outer edge. Four sensors are placed at the base of each pillar, centered under the edge, as shown in Fig. 2, to achieve maximum response. The data from each element are combined to deduce the applied force vector on the corresponding pillar, in three dimensions.

In addition to obtaining triaxial force information, the PDMS pillars provide compliance and surface friction similar to those of human skin.

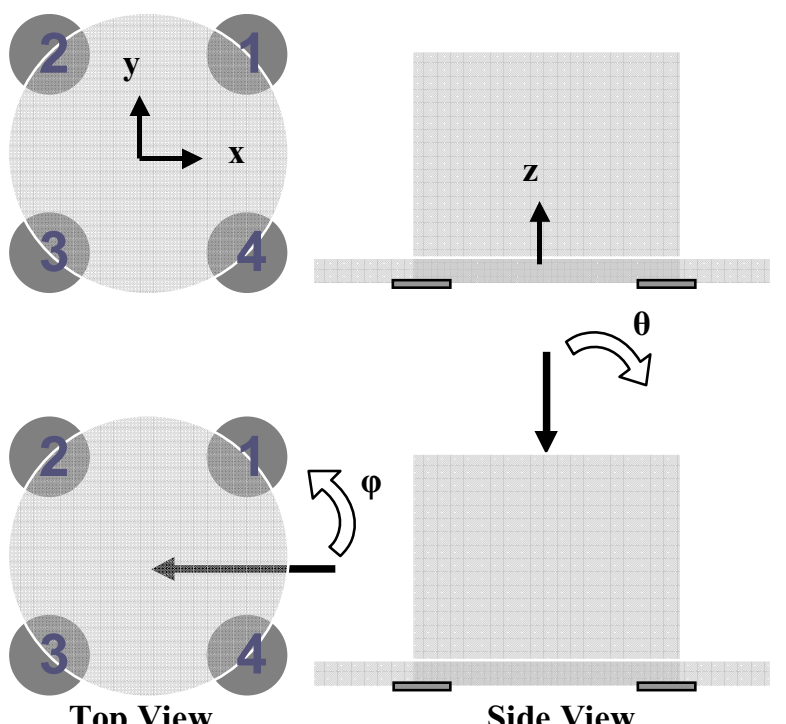

Top View

Side View

Fig. 2 Top and side sketches of a single pillar. The darker, numbered regions are sensor locations. Fig. $2 \mathrm{a}$ and $2 \mathrm{~b}$ show two different coordinate systems

\section{PILLAR THEORY}

In the Cartesian coordinate system shown in Fig. 2a, the components of an applied force vector can be determined with simple combinations of the sensor voltages. For example,

$$
\begin{aligned}
& F_{x} \propto V_{1}+V_{4}-V_{2}-V_{3}, \\
& F_{y} \propto V_{1}+V_{2}-V_{3}-V_{4}, \\
& F_{z} \propto V_{1}+V_{2}+V_{3}+V_{4},
\end{aligned}
$$

where $V_{n}$ is the voltage output by sensor $n$. These equations, however, require that $\mathrm{V}_{\mathrm{n}}$ is allowed to be negative. If, for example, there is a large force directed solely in the $\mathrm{x}$ direction, the pillar deforms towards sensors one and four and up and away from sensors two and three. Since the piezoresitive sensor technology only responds to compression, $\mathrm{V}_{2}$ and $\mathrm{V}_{3}$ read zero and do not provide the necessary negative voltage to provide accurate $F_{x}$ in (1). In addition, $F_{z}$ would not be zero because $V_{2}$ and $V_{3}$ are not negative to cancel out the positive magnitude of $\mathrm{V}_{1}$ and $\mathrm{V}_{4}$.

\section{A. Triaxial Force Discernment Algorithm}

In order to overcome this limitation, we developed an algorithm that does not require "useable" (i.e. strictly positive) data from all four sensors. By switching from Cartesian coordinates, as used in (1), to spherical coordinates, as shown in Fig. 2b, the voltage at each sensor is modeled as

$$
V_{n}=F \cdot k\left(\cos (\theta)-\sin (\theta) \cos \left(\frac{\pi}{4}(2 n-1)-\phi\right)\right),
$$

where $F$ is the magnitude of the applied force, and $\theta$ and $\varphi$ represent the direction of the applied force. $\varphi$ is the angle in the plane of the top surface of the pillar, and $\theta$ is the angle measured from the normal of the pillar's top surface. $\mathrm{k}$ is a constant, which depends on physical characteristics of the pillar such as height and width relative to the sensor locations and Poisson's ratio and Young's modulus. This model describes the relationship between the applied force, in spherical coordinates, to the reading on each of the sensors. Given the applied force we can find the resulting sensor voltages, or given the sensor voltages we can solve a system of equations to find the applied force.

Using this model we can examine the range of directions of the applied force that will give us reliable sensor voltages. Furthermore we can stratify all possible forces into separate regions where specific sensors have usable data. This stratification is central to the pillar algorithm because multiple techniques will be used to solve for the applied force and each technique is only valid in a specific region.

As mentioned above, $V_{n}$ is never negative for our sensors. Therefore, it is important to pay attention to the cases when $V_{n}$ in (2) becomes negative. To do so, we defined boundary lines that are defined as

$$
\theta=\cot ^{-1}\left(\cos \left(\frac{\pi}{4}(2 n-1)-\phi\right)\right),
$$

where $V_{n}$ is zero. When theta is greater than this value, sensor n provides "unusable" data.

Boundary lines for all four sensors are plotted in Fig. 3. The four boundary lines divide this space into nine sections. These sections are organized into four higher-level regions. In Region 1, all four sensors give usable outputs. In Region 2, 


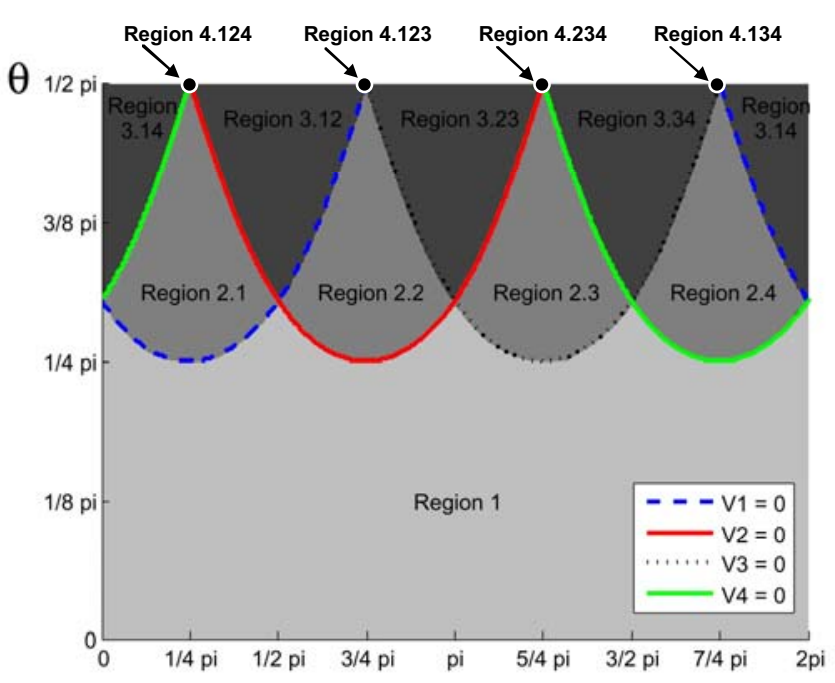

Fig. 3 Plots of the $V_{n}=0$ boundary where a sensor's output becomes unusable. Below each curve the reading is strictly positive and usable, above the curve, the reading is unusable.

three of the four sensors have usable outputs and the subdivisions represent sections in which a particular sensor's output is unusable. For example, in Region 2.1, sensor one's output is unusable. In Region 3, two of the four sensor readings are unusable, and again, the subdivisions indicate the sensors that are unusable. Region 4 , indicated by the four black dots along the top of Fig. 3, is comprised of the four special cases in which three of the four sensors have unusable data. In each of these high-level regions a different technique is used to determine the magnitude and direction of the applied force.

1) Region 1: To solve for all components of the applied force, in region 1 , where all sensors have usable readings, we first eliminate $\mathrm{F}$ and $\mathrm{k}$ by taking the ratio, $\mathrm{R}_{\mathrm{ij}}$, of equation (2) for two sensors. This ratio is

$$
R_{i j}=\frac{\cos (\theta)-\sin (\theta) \cos \left(\frac{\pi}{4}(2 i-1)-\phi\right)}{\cos (\theta)-\sin (\theta) \cos \left(\frac{\pi}{4}(2 j-1)-\phi\right)} \text {, where } R_{i j}=\frac{V_{i}}{V_{j}},
$$

where $\mathrm{i}$ and $\mathrm{j}$ represent the two sensor numbers. Equation (4) gives $\theta$ as a function of $V_{i}, V_{j}$, the voltage on sensors $i$ and $j$, and $\varphi$ :

$$
\tan (\theta)=\frac{R_{i j}-1}{R_{i j} \cos \left(\frac{\pi}{4}(2 j-1)-\phi\right)-\cos \left(\frac{\pi}{4}(2 i-1)-\phi\right)}
$$

If we combine (5) with the same equation using the other two sensors, $\mathrm{k}$ and 1 , we can find an equation for $\varphi$ as a function of just the sensor voltages,

$$
\begin{gathered}
\tan (\phi)=\frac{R_{i j} R_{k l}[\cos (L)-\cos (J)]+R_{i j}[\cos (J)-\cos (K)]+R_{k l}[\cos (I)-\cos (L)]+[\cos (K)-\cos (I)]}{R_{i j} R_{k l}[\sin (J)-\sin (L)]+R_{i j}[\sin (K)-\sin (J)]+R_{k l}[\sin (L)-\sin (I)]+[\sin (I)-\sin (K)]}, \\
\text { where CAPITAL }=\left(\frac{\pi}{4}(2 * \text { lowercase-1) }),\right.
\end{gathered}
$$

and $R_{i j}$ and $R_{k l}$ are the ratios of sensor voltages, as shown in (4). Finally by assigning specific sensor numbers, (e.g. $\mathrm{i}=1, \mathrm{j}$ $=3, \mathrm{k}=2,1=4)$, (6) becomes

$$
\tan (\varphi)=\frac{V_{1} V_{2}-V_{3} V_{4}}{V_{1} V_{4}-V_{2} V_{3}}
$$

Using equations (2), (5) and (7) we can solve for all components of the applied force vector.

2) Region 2: If one of the sensors has an unusable output, as is the case in Region 2 of Fig. 3, we can find a direct solution for all components of the applied force. Using usable sensors $\mathrm{i}, \mathrm{j}$, and $\mathrm{k}$, one can create two equations of the form of equation (5), one using $\mathrm{R}_{\mathrm{ik}}$ and $\mathrm{R}_{\mathrm{jk}}$. In that situation, (6) becomes

$$
\tan (\phi)=\frac{R_{i k}[\cos (K)-\cos (J)]+R_{j k}[\cos (I)-\cos (K)]+[\cos (J)-\cos (I)]}{R_{i k}[\sin (J)-\sin (K)]+R_{j k}[\sin (K)-\sin (I)]+[\sin (I)-\sin (J)]}
$$

$\mathrm{I}, \mathrm{J}$, and $\mathrm{K}$ are proportional to $\mathrm{i}, \mathrm{j}$, and $\mathrm{k}$, as shown in part two of (6). Supplying specific values for the sensor numbers, in this case, generates four equations, one for each subsection of Region 2:

$$
\begin{aligned}
& \text { Region 2.1: } \tan (\varphi)=\frac{V_{2}-V_{3}}{V_{3}-V_{4}} ; \text { Region 2.2: } \tan (\varphi)=\frac{V_{4}-V_{1}}{V_{3}-V_{4}} ; \\
& \text { Region 2.3: } \tan (\varphi)=\frac{V_{4}-V_{1}}{V_{1}-V_{2}} ; \text { Region 2.4: } \tan (\varphi)=\frac{V_{2}-V_{3}}{V_{1}-V_{2}} .
\end{aligned}
$$

3) Region 3: This is the only case where we are unable to provide a direct solution. When two of the sensors have usable outputs, explicitly solving for the three components of the force is not possible. However, given the two sensors that have usable readings, we know the general range of values that $\theta$ and $\varphi$ must have because they are confined to a specific region in Fig. 3. Within each region, the size of the uncertainty range is $\pi / 2$ radians in $\varphi$ and approximately $3 \pi / 16$ in $\theta$. This uncertainty can be reduced further using equation (5), which gives $\theta$ as a function of $\mathrm{R}_{\mathrm{ij}}$, a ratio of the two sensor values with usable readings, and $\varphi$. Since we know $\mathrm{R}_{\mathrm{ij}}$, this equation defines an explicit relationship between $\theta$ and $\varphi$. Fig. 4 shows a few examples of the contours traced by this relationship for different values of $R_{i j}$. By solving for the intersection of these contours with the sensors boundary

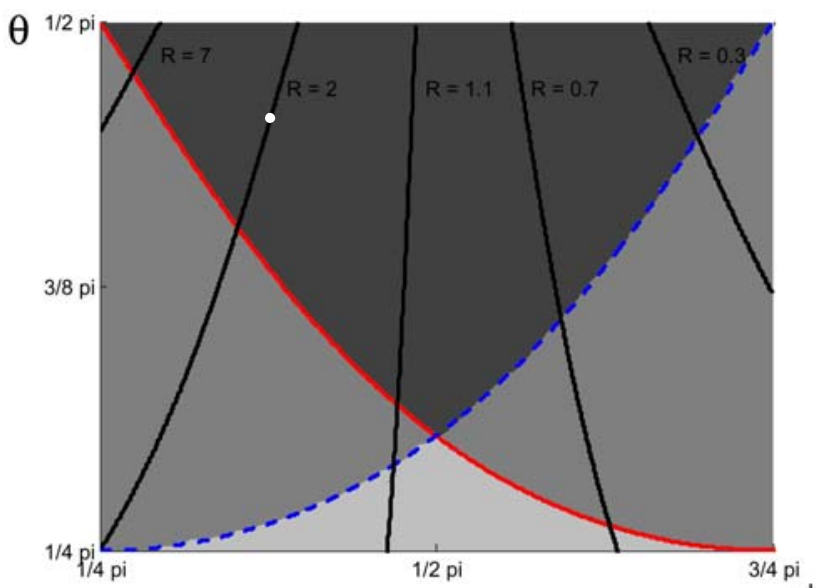

Fig. 4 Contours of equation (5) for various values of R, plotted over $\phi$ region 3.12 from Fig. 3. The white dot on the $R=2$ contour is half way between the upper and lower bounds of the region. 


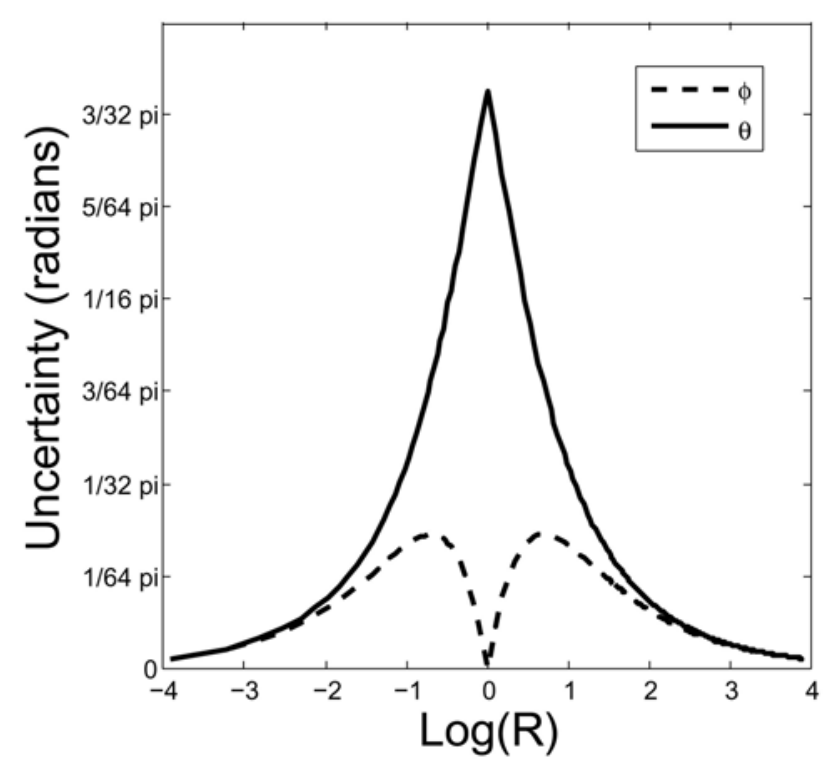

Fig. 5 Uncertainty in $\theta$ and $\varphi$, plotted as a function of the logarithm of the ratio between two sensor voltages

curves and $\theta=\pi / 2$, the maximum and minimum values of $\theta$ and $\varphi$ are identified.

While the possible range of $\theta$ and $\varphi$ is narrowed, there is still an uncertainty in the angles. To further reduce the uncertainty we can choose the point on the contour of $\mathrm{R}_{\mathrm{ij}}$ that is halfway between the boundaries of the corresponding region. This is illustrated by the white dot on the $\mathrm{R}=2$ contour in Fig. 4. This approach cuts the maximum uncertainty by a factor of two. Fig. 5 shows this uncertainty as a function of $\mathrm{R}_{\mathrm{ij}}$. This figure is valid for any pair of adjacent sensors with reliable outputs. The maximum uncertainty in $\theta$ occurs at $R_{i j}=1$ when the uncertainty in $\varphi$ is at a minimum. As $R_{i j}$ gets large or small the uncertainty in both parameters diminishes.
4) Region 4: Finally, in the rare case that only one sensor has a useable reading, we are restricted to one of the four single points in Fig. 3. By knowing which of the four sensors is providing the usable signal, we are able to identify the exact $\theta$ and $\varphi$. For example, if sensor four is the only usable sensor, then the solution is Region 4.123 where $\theta=\pi / 2$ and $\varphi=3 \pi / 4$.

\section{Algorithm Summary}

In the situations where zero, one, or three sensors gives unusable data, all force components can be solved explicitly. That is, for the majority of the possible applied forces, direct solutions for all components of that force exist. The forces are ambiguous only when two sensors are unusable. In this situation, $\theta$ and $\varphi$ cannot be solved explicitly but are bounded within known values with a small uncertainty range. The maximum uncertainties for $\theta$ and $\varphi$ are 0.32 radians and 0.07 radians respectively.

\section{Pillar Testbed}

In order to validate the triaxial force discernment algorithm, a pillar testbed was developed. Fig. 6 shows two views of our testbed that incorporates a force probe positioning system (FPPS). The sensors are Flexiforce sensors from Tekscan and the pillar is made from polydimethylsiloxane (PDMS). This testbed is nearly two orders of magnitude larger than the eventual size of a pillar in the high density array. The size was chosen based on the size of the Flexiforce sensors, which are large, single-sensor versions of the Tekscan's piezoresitive technology. A calibrated Omega ${ }^{\mathrm{TM}}$ force probe with the force resolution of $0.1 \mathrm{~N}$ is mounted to a 5-DOF positioning system. A shaft is attached to the force probe and the tip of this shaft can be placed in contact with the pillar for force application. A lead

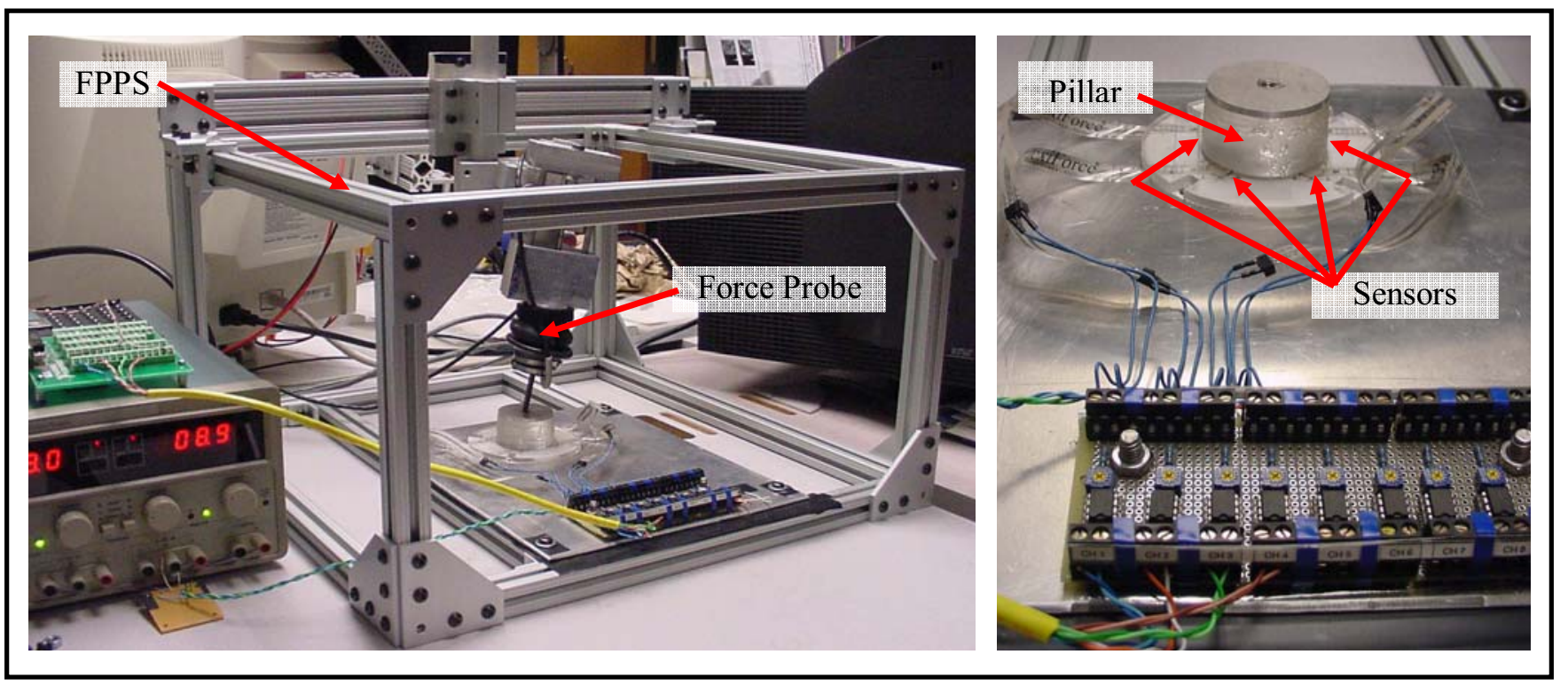

Fig. 6 Two views of the pillar testbed. LEFT: The overall layout including FPPS and force probe. RIGHT: Detailed layout of pillar testbed including pillar, sensors, and sensor excitation electronics. 


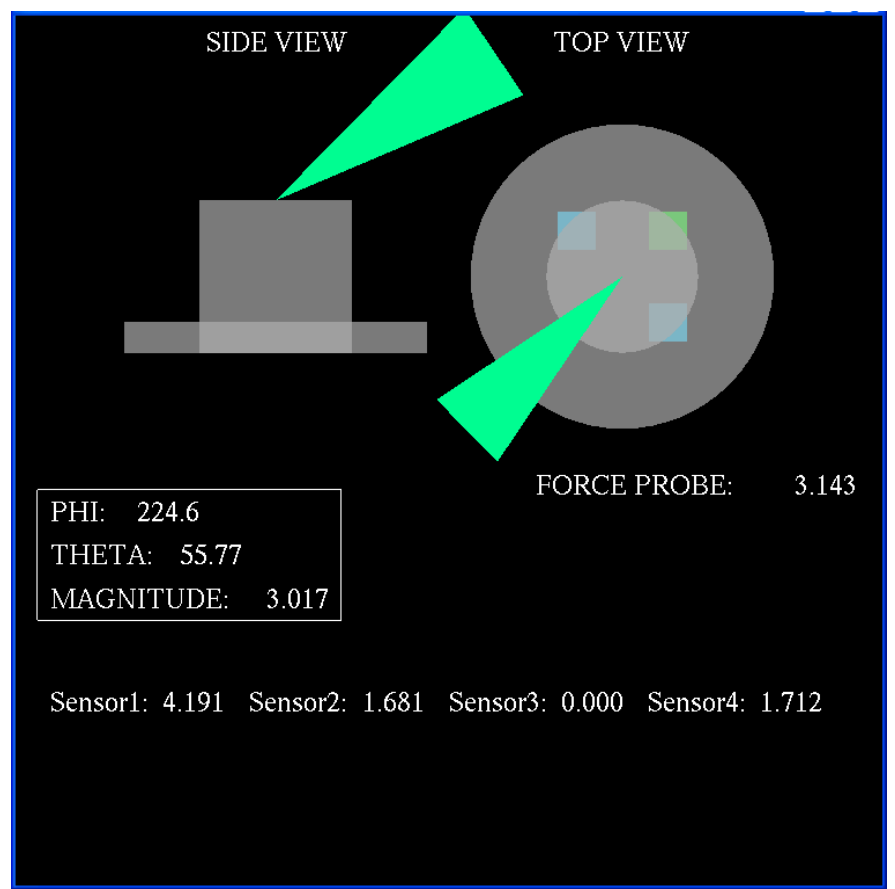

Fig. 7 Pillar testbed interface.

screw mechanism allows the user to change the magnitude of the applied force. Attached to the top of the pillar is a metal plate which disperses the applied force over the entire surface of the pillar. With this system a large range of directions and magnitudes of force can be applied to the pillar.

A data acquisition system is used to digitize and store the output of the four sensors. Using the triaxial force discernment algorithm, an interface, shown in Fig. 7, displays the calculated angles and magnitude of the applied force based on the sensor inputs.

\section{RESULTS}

Using the FPPS, multiple forces were applied to the pillar. Data was collected by adjusting the FPPS to a specific angle and then applying forces between $10 \mathrm{~N}$ and $100 \mathrm{~N}$ at $10 \mathrm{~N}$ intervals. Table II displays mean, standard deviation, and mean error values calculated over this range of forces. One example for Regions 1, 2 and 3 is shown. In Region 3, the difference between the ground truth and the mean values for $\theta$ and $\varphi$ are two to three times larger then the same differences for Regions 1 and 2. This is an expected result because in Region 3 only two sensors have usable outputs and the results are uncertain. In Region 1, the standard deviation in $\theta$ is large compared to the other regions. This result is primarily caused by one of the sensors remaining zero for small forces. Based on the number of usable sensors, the algorithm determined that the applied force fell into Region 2 and these data points were calculated with incorrect assumptions. This situation requires additional checks that will be implemented in future versions of the algorithm.

TABLE II

Comparison of Ground Truth and Experimental Results for $\theta$ and $\varphi$

\begin{tabular}{|c|c|c|c|c|}
\hline & $\begin{array}{c}\text { Ground } \\
\text { Truth }\end{array}$ & Mean & $\begin{array}{l}\text { Standard } \\
\text { Deviation }\end{array}$ & Mean Error \\
\hline \multicolumn{5}{|c|}{ Region 1} \\
\hline$\varphi$ & 2.094 & 2.043 & 0.093 & 0.084 \\
\hline$\theta$ & 0.471 & 0.551 & 0.240 & 0.177 \\
\hline \multicolumn{5}{|c|}{ Region 2} \\
\hline$\varphi$ & 3.927 & 3.893 & 0.152 & 0.084 \\
\hline$\theta$ & 0.977 & 0.926 & 0.113 & 0.0735 \\
\hline \multicolumn{5}{|c|}{ Region 3} \\
\hline$\varphi$ & 5.760 & 5.890 & 0.032 & 0.130 \\
\hline$\theta$ & 1.222 & 1.417 & 0.013 & 0.196 \\
\hline
\end{tabular}

The uncertainty in the ground truth is approximately 0.05 radians. The main contributions to this uncertainty are the 0.04 radian accuracy of the FPPS and a small amount of play in the FPPS that results in small changes in the angle of the force probe as the force increases. The data in Regions 1 and 2 are within or nearly within this range of uncertainty.

The uncertainty in the angles also propagates into the
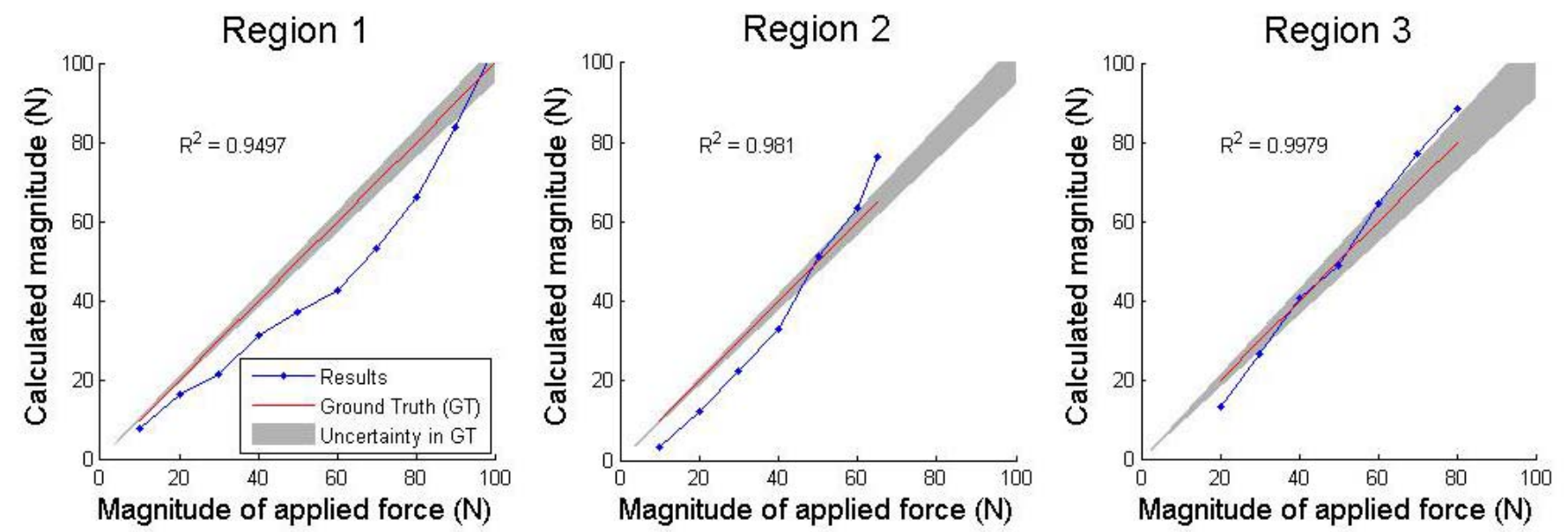

Fig. 8 Calculated magnitude as a function of the ground truth force magnitude. The shaded grey areas represent the uncertainty in the ground truth force that results from uncertainty in the angles $\theta$ and $\varphi$ 
magnitude calculations. Fig. 8 shows magnitude plotted as a function of the applied force. The red lines represent the ground truth values and the grey patches show the effect of the uncertainty in $\theta$ and $\varphi$ on the magnitude calculations.

\section{DISCUSSION AND FUTURE WORK}

We introduced a new flexible, high resolution sensing array design, as well as a triaxial force discernment algorithm. Together they comprise the first system to integrate three critical characteristics of artificial skin: high density, flexibility, and triaxial force discernment. The pillar testbed results show that the pillar design and the force discernment algorithm are capable of resolving all three components of an applied force. The next step is to miniaturize the design and begin working with multiple pillars. Several critical issues will be addressed during testing of the high resolution design. In particular, stress due to bending while conforming to curved surfaces must be accounted for through calibration. Also due to the flexible pillar layer, sensing elements may be affected by deformation of neighboring pillars. Minimizing this crosstalk may be achieved by minimizing the thickness of skin between pillars. Furthermore, as has been noted, the main limitation of the pillar design is the ambiguity of the data when only two sensors provide usable data. We plan to investigate whether a large array of pillars can help resolve ambiguity by sharing information between neighboring sensor elements.

\section{ACKNOWLEDGMENTS}

This work was funded in part by grants NSF 0423546 and NIH R21EB005967.

\section{REFERENCES}

[1] D. Yamada, T. Maeno, Y. Yamada, "Artificial finger skin having ridges and distributed tactile sensors used for grasp force control", J. of Robotics and Mechatronics, vol 14, No. 2, pp. 140-146, 2002.

[2] T. Papakostas, J. Lima, M. Lowe, "A large area force sensor for smart skin applications," Sensors, Proceedings of IEEE, vol. 2, pp. 1620-1624, 2002.

[3] B. Kane, M. Cutkosky, G Kovacs, "A traction stress sensor array for use in high-resolution tactile imaging", J. of Micromechanical Systems, vol. 9, No. 4, December 2000.

[4] R. Liu, L. Wang, D. Beebe, "Progress towards a smart skin: fabrication and preliminary testing", Engineering in Medicine and Biology Society, Proceedings of IEEE, vol. 20, No. 4, 1998.

[5] M. Hakozaki and H. Shinoda, "Digital Tactile Sensing Elements Communicating through Conductive Skin Layers", International Conference on Robotics and Automation, Proceedings of IEEE, vol 4. pp. 3813-3817, 2002.

[6] http://www.tekscan.com

[7] R. Howe, M. Cutkosky, "Dynamic Tactile Sensing: Perception of Fine Surface Features with Stress Rate Sensing", IEEE Trans. On Robotics and Automation, vol. 9, No. 2, April 1993.

[8] P.Dario and D. de Rossi, "Tactile sensors and the gripping challenge", IEEE Spectrum, pp. 46-52, August 1985. 